\title{
Correspondence
}

\section{Antimicrobial Storage and Antibiotic Resistance}

To the Editor: The astounding scenario responsible for emergence and dissemination of antimicrobial resistance $^{1}$ is operational in poor and richer communities alike. To address the issue universally, it is important to appreciate the recommendations for storage of antimicrobials. They require constant storage at controlled temperatures ranging from sub-zero to $10^{\circ} \mathrm{C}$ to $25^{\circ} \mathrm{C}$. ${ }^{2}$ They are inadvertently subjected to harsh environment before administration to patients with a reduced effective potency. That is not an exclusive episode in poor countries. During the 1995 heat wave in Chicago, the temperature was $40^{\circ} \mathrm{C}$, but the heat index, an estimate of evaporative and radiative transfer of heat, was $48.3^{\circ} \mathrm{C} .^{3}$ Different agencies collaborated to minimize the harmful effects of heat on humans: the therapeutics including antimicrobials were ignored. Furthermore, electrical appliances are essential toward maintenance of constant temperature. Although erratic power supply is a rule rather than an exception in poor countries, the picture during the Hurricane Katrina in New Orleans during 2005 was shocking. The prolonged power cut was accompanied with auxiliary generators running out of fuel. ${ }^{4}$ Prospective research in the United States or elsewhere to establish the factors responsible for antimicrobial failures $^{1}$ should investigate their storage practices in warehouses, physician's offices, and pharmacy and non-pharmacy sites. In the interim phase, the storage specifications on antimicrobial containers should as well bear distinct symbols indicating the recommended storage temperature. That should not be an insurmountable task because distinct marks are mandatory for containers of poisons, radioactive materials, and inflammables and are in universal usage.

The quality of antimicrobials in actual usage by any patient would be best measured at the point of usage. Rather than multistep tests with costly equipment, simpler assays without highly trained personnel or sophisticate equipment are needed on a priority basis. Fiscal input toward improved versions of field and simpler assays for paracetamol or rapid spectrophotometer analysis for antimalarial formulations ${ }^{5,6}$ directed toward antimicrobials would be cost-effective.

Planta's ${ }^{1}$ recommendation to individual hospitals and health care centers to monitor antimicrobial susceptibility of prevailing isolates is commendable and worth emulation. A syndrome-wise categorization of efficient oral and parenteral antimicrobials would interest the clinician toward their rational selection of antimicrobials while awaiting laboratory results on clinical samples. At a 140bed private, tertiary care, multispecialty hospital in Delhi, a syndrome-wise categorization has been operational since $2004 .^{7}$ Rather than relying on fourth-generation cephalosporin in aggressive infection, hospitalized cases would be offered alone or in combination from meropenem, piperacillin-tazobactam, amoxicillin-clavulanic acid, and amikacin. ${ }^{8}$

Subhash C. Arya

Sant Parmanand Hospital, Delhi, India subhashji@hotmail.com

Nirmala Agarwal

Sant Parmanand Hospital, Delhi, India

\section{References}

1. Planta MB. The role of poverty in antimicrobial resistance. J Am Board Fam Med 2007;20:533-39.

2. Physicians' Desk Reference. 61st ed. Montvale (NJ): Medical Economics Company; 2007.

3. Centers for Disease Control and Prevention (CDC). Heatrelated mortality-Chicago, July 1995. MMWR Morb Mortal Wkly Rep 1995;44:577-9.

4. Dalton R. Health centres and labs left reeling by Katrina. Nature 2005;437:177.

5. Shakoor O, Taylor RB, Beherns RH. Assessment of the incidence of substandard drugs in developing countries. Trop Med Int Hlth 1997;2:839-45.

6. Morris K. Malaria death due to fake drugs deemed 'manslaughter'. Lancet Infect Dis 2006;6:470.

7. Arya SC, Agarwal N, Solanki BS, Agarwal S. Use of cefepime for the treatment of infections caused by extended-spectrum beta-lactamase-producing Klebsiella pneumoniae and Escherichia coli. Singapore Med J 2007;48: $600-1$.

8. Arya SC, Agarwal N, Agarwal S. Straightforward representation of antimicrobial chemotherapeutics susceptibility profiles in a private tertiary care hospital. J Hosp Infect 2005;51:333-5.

doi: $10.3122 / \mathrm{jabfm} .2008 .02 .070233$

\section{Response to Issue of Antimicrobial Storage}

To the Editor: Arya writes that improper storage of antimicrobials may affect their potency and contribute to resistance. This is an important issue and is a known issue with respect to vaccines. However, Arya starts with some faulty and unsubstantiated premises.

First, very few commonly used antibiotics in the outpatient setting in the Unites States require constant storage at subzero temperatures.

Then the author fails to substantiate the statement "they are inadvertently subjected to harsh environment before administration to patients...." The author also fails to substantiate the supposition that a harsh environment leads to "a reduced effective potency."

The author implies that the 1995 heat wave in Chicago affected the quality of medications, especially antimicrobials. I was present in Chicago as a medical student during this heat wave. What the author fails to realize is that hospitals and pharmacies are air-conditioned, very few areas lost power, and 
hospitals have back-up generators in case of power loss, so the heat wave did not affect medications stored in hospitals or pharmacies. It is doubtful that storage issues are a significant contributor to antimicrobial resistance and failure in the United States, and research within the United States would be more useful investigating other areas.

Because power is erratic in developing countries, and air conditioning may be unaffordable to many facilities, storage issues and resultant antimicrobial failure is more likely to occur in those countries. Certainly in those countries, such research should be undertaken and the author's suggestion of labeling medications with storage specifications may prove useful.

Degradation of antimicrobials due to improper storage in developing countries can indirectly affect the United States, because US residents may be purchasing these foreign-made and improperly stored drugs. It may be possible that the lack of or reduced potency of active ingredient detected in shipped foreign-made drugs mentioned in my paper is due, in part, to improper storage, rather than just improper manufacture.

Testing medication potency at point of usage is not feasible as one would need to develop an assay for every antimicrobial, and this assay would need to be available in every town or village in each country. The author fails to substantiate the statement that developing this assay "would be cost-effective."

Two of the suggestions mentioned in my paper can help counteract this storage issue. First is eliminating over-thecounter dispensation of antimicrobials and limiting them to licensed pharmacies, whose storage mechanisms are monitored by the government. Second is the regulation of the manufacture, storage, and distribution of drugs to ensure their potency at the point of distribution to hospitals and pharmacies. Again, the feasibility and costs of these suggestions are yet to be determined.

In the last paragraph, the author's suggestion of syndromewise categorization on an individual community/hospital basis is interesting and warrants further consideration and possible emulation. However, there needs to be research as to whether this system implemented in 2004 has resulted in reduced resistance in the surrounding community, or at the very least, in nosocomial isolates in the hospital.

As a final note, my paper could not encompass all the issues that may be contributing to resistance in the United States I would also suggest research into the effects of antimicrobial use in farm animals, as well as the appearance of antimicrobials in groundwater/rivers/streams resulting from the disposal of leftover antibiotics into sewage systems (ie, "flushing them down the toilet").

\section{Addendum}

On November 1, 2007, the United States Government Accountability Office (GAO) issued a report ${ }^{1}$ stating:

1. Approximately 3000 foreign establishments are registered to market drugs in the United States in 2007, but 6800 foreign establishments may actually import drugs into the United States.

2. The FDA may only inspect $7 \%$ of foreign establishments in a given year.

3. In 2007, China and India had more establishments registered to manufacture drugs for the US market than any other country.
This raises the possibility that generic antimicrobials, sold by discount pharmacies and mail order programs, may originate from a foreign country whose manufacture and storage practices are unregulated and may result in suboptimal potency of antimicrobial drugs dispensed in the United States.

\section{References}

1. Drug Safety: Preliminary Findings Suggest Weaknesses in FDA's Program for Inspecting Foreign Drug Manufacturers, Statement of Marcia Crosse, Director Health Care, Testimony Before the Subcommittee on Oversight and Investigations, Committee on Energy and Commerce, House of Representatives, United States Government Accountability Office; 2007 Nov 1; 1 to 28.

Margaret Planta, MD

Sutter Medical Group, Roseville, CA mplanta@pol.net

doi: 10.3122/jabfm.2008.02.070243

\section{African Family Medicine}

To the Editor: "Shifting the world's paradigm to 'primary care access for all" implies that family medicine provides an excellent means to facilitate this shift, even in Africa. Indeed, the evidence for the efficacy of primary care systems in improving health in the industrialized world is clear. Montegut ${ }^{1}$ reviews 6 "practice characteristics" that are related to better health outcomes, and Beasley et $\mathrm{al}^{2}$ describe 4 of them in some detail. The implication is that if primary care generally, and family medicine specifically, is to lead to better health outcomes, it should at least comprise these characteristics. So how closely does this fit with family medicine as it is developing in Africa?

It is difficult to say whether these 6 characteristics will be as beneficial in Africa; studies need to be done in Africa asking this question. But my experience in a new family medicine training program in Kenya, together with at least 1 continent-wide survey (so far unpublished), suggest that these particular characteristics are not always the first priorities for African family medicine.

First contact care and "gate-keeping," for example, is not a common characteristic of African family medicine; this is often done by nurses or physician assistant-level providers. ${ }^{3}$ Longitudinal care is very difficult where chronic disease is uncommon, and the majority of patients come for acute episodic care. Comprehensive care is a goal, but African family physicians do not rank "preventive medicine" as their first priority. Rather, they are concerned with being good generalists, and in most African settings, this involves not only inpatient care but also major emergency surgery.

Of course it may be that these 6 characteristics could be conducted by other parts of the primary care systems in Africa, ultimately leading to improved health outcomes. Yet I suspect that in countries where half of the people live on less than a dollar a day, where roads are poor and transport expensive, where people do not have habits of "check-ups" or daily medicine-taking, these 6 characteristics may not be as important in improving 